\title{
Palliative Care - oder «Sterben ist eine Kunst»
}

\section{Jean Martin}

Dr. med., Mitglied der Redaktion

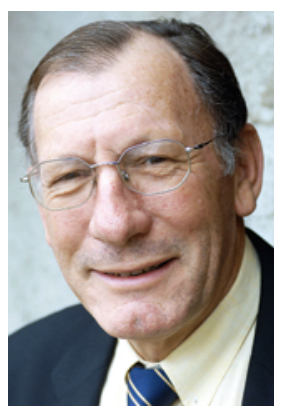

Am 19. und 20. September hatte der Verband Curaviva seinen Fachkongress Alter 2017 (7. Ausgabe) in Montreux. Es war ein Erfolg mit 1300 Teilnehmenden. Parallel dazu fand auch die «Global Ageing Conference» des Global Ageing Network statt, an der sich ein interessiertes Fachpublikum aus dutzenden Ländern begegnete. $\mathrm{Zu}$ hören waren «Keynote Speeches» bekannter Referentinnen und Referenten aus unterschiedlichen Disziplinen, darunter alt Bundesrätin Micheline Calmy-Rey, der international bekannte Starkoch Anton Mosimann und der charismatische Professor der Genetik, Dr. Markus Hengstschläger aus Wien.

\section{Der Patient selbst sollte das Orchester leiten!}

Eine Parallelveranstaltung mit dem Titel «Sterben ist (k)eine Kunst» fand mein besonderes Interesse. Die Verantwortlichen des Kompetenzzentrums Pflege und Gesundheit (KZU) in Bassersdorf (ZH) und des Altersund Pflegeheims Erlenhaus in Engelberg (OW) stellten ihre Praktiken in der Palliativen Care und im Umgang mit Sterbenden vor. Hier möchte ich auf den Beitrag von Theres Meierhofer-Lauffer, der Leiterin des Heims Erlenhaus, eingehen. Sie ist gleichzeitig auch Mitglied der Arbeitsgruppe Palliative Care ihres Kantons. Ihre Einrichtung beherbergt 50 Bewohnerinnen und Bewohner, von denen jährlich 40 Prozent versterben (gilt auch für das oben genannte KZU). Eine grosse Herausforderung! Theres Meierhofer nennt vier Themen: interdisziplinäre Zusammenarbeit, Begleitung der Angehörigen, Rituale beim Sterben, Symptomlinderung-und bezieht sich dabei wiederholt auf die Best-Practice-Bigorio-Empfehlungen von Palliative.ch.

Sie ist der Ansicht, dass unser Handeln auf Beziehungen basiert und dabei getreu der Devise «Geborgen im Leben - Geborgen im Sterben», die jeweiligen Ressourcen der Betroffenen, ihre Interessen und das gemeinsame Leben berücksichtigt. So ist beispielsweise das Erlenhaus-Team bestrebt, zusammen mit den Patienten deren Geschichte ins Gedächtnis zurückzurufen und darüber zu sprechen, was sie im Haus erleben. Diese Gespräche zeitigen eine positive Wirkung auf die Symptome - natürlich begleitend zum Einsatz anderer Mittel.
Ausserdem verweist Theres Meierhofer-Lauffer auf die Bedeutung von Ritualen. Dabei geht es nicht darum (wie vielleicht früher der Fall), das Ableben eines Menschen im Alters- und Pflegeheim so diskret wie möglich durch die Hintertür zu behandeln. «Wir gehen durch den Haupteingang», sagt sie und beschreibt eine besondere Zeremonie, wenn der Sarg das Haus verlässt, in Gegenwart der Mitarbeiter. Es gibt ein Treffen mit den Bewohnerinnen und Bewohnern. Man spricht über den Gegangenen und darüber, was der Weggang für die Zurückgebliebenen bedeutet.

Die Angehörigen erhalten viel Aufmerksamkeit und Zuwendung - sogar ein Frühstück am Morgen nach dem Todestag. Die Lebensumgebung ist ländlich, mit lokalen Gewohnheiten. Alle kennen sich. Meierhofer erklärt, dass das Team so die Möglichkeit hat, den Kontakt zu den Angehörigen flexibel zu gestalten und beispielsweise an allfällige Schwierigkeiten in einer Familie anpassen zu können (was sich in einem urbanen Milieu offenkundig weniger leicht realisieren lässt). Am Ende des ganzen Prozesses trifft sich das Team und erörtert die Frage, ob während des gesamten Aufenthalts der Person bis zu deren Ende alles richtig gemacht wurde.

Die Aus- und Weiterbildung aller Akteure im Bereich Palliative Care ist von grundlegender Bedeutung. Sie richtet sich nach dem Leitsatz «Die Begleitung im Leben und im Sterben ist unser Kerngeschäft» (core business). Bei dieser Ausbildung geht es darum, dem Anderen Aufmerksamkeit zu schenken, Werte (eigene und die des Anderen) zu schätzen und nach ihnen zu handeln. Und es geht um die Frage nach professionellen und individuellen Verhaltensweisen. Auch die spirituelle Komponente hat ihren Platz. Für den Stiftungsrat der Einrichtung wurden Schulungen organisiert (an denen unter anderem auch die Frage der Sterbehilfe erörtert wurde). Und schliesslich zum Thema interdisziplinäre Zusammenarbeit: Was mit und um den Patienten selbst geschieht, erinnert an ein Orchester, wo jeder seine eigenen Noten zu spielen hat. Die Referentin erklärt, dass - soweit möglich - der Patient selbst als Orchesterleiter agieren sollte und die Fachkräfte ihn in diesem Ziel unterstützen und es ihm ermöglichen sollten! 